Computer Science

Dec 2014

\title{
Search-and-Rescue Algorithm: Detecting Access Holes in Disaster Rubble
}

Soon after our species began constructing buildings thousands of years ago we also had to start dealing with our homes and other buildings collapsing - whether the result of natural forces such as earthquakes or typhoons, or the effect of human causes such as sabotage, warfare or just plain shoddy workmanship. A grave consequence of structural collapse is the fact that people often find themselves trapped and injured within rubble, all too often dying before they can be found and extricated.

\section{All about Holes}

An MSc student in Ryerson's Department of Computer Science, Christopher Kong is the lead author of a recently published paper that documents advances he and fellow members of the Network-Centric Applied Research Team (NCART) have made in using computer technology to assist first responders in such situations. In this work, they are the first to have developed a system to identify access holes in collapsed structures using computation rather than visual surveillance. As NCART's director, Dr. Alex Ferworn observes, the system addresses the "pernicious problem of time-to-rescue. On average, people trapped under rubble survive about 48 hours; therefore, finding victims quickly is very important."

In support of that goal, Christopher says his research is "all about holes." As he explains, "I devise algorithms that analyze video and distance data collected from a sensor that's mounted under an unmanned aerial vehicle (UAV) - or drone - flying over rubble. The goal is to detect access holes that rescuers can use to enter in their search for survivors." As Christopher and his co-authors explain in their paper, "We concentrate not on the identification of rubble but rather on identifying its absence."

\section{Minimizing Risk, Maximizing Effectiveness}

Christopher and his NCART colleagues refer to the broad field in which they work as "computational public safety," which Dr. Ferworn describes as "the application of computational resources, theory and practice in support of and improvements to public safety processes."

Urban search and rescue (USAR) personnel face considerable risks and challenges in their search for building-collapse survivors. To begin with, there is the inherent instability of rubble, which can make physical searching for holes potentially lethal. "Using a remotely operated drone," Christopher notes, "removes humans from this dangerous task."

In addition, USAR personnel normally need to visually inspect large swathes of debris, a task that can lead to cognitive fatigue. The system Christopher and his colleagues are developing, however, eliminates that burden by directing rescuers' attention towards "high-probability locations." A final advantage of their UAV-mounted system is the capability of investigating regions beyond the line of sight for human searchers traversing uneven rubble fields.

\section{Real-world Relevance and Testing}


"One of the best aspects of my research has been knowing that it has direct, real-world applications that may, one day, help to save lives," Christopher says. "Another exciting dimension has been working with tele-operated robots, multicopter flying drones and 3D printers." Christopher and his NCART team-mates have also had the opportunity to conduct their experiments and collect data at the Ontario Provincial Police's Urban Search and Rescue Team facility in Bolton, Ontario, as well as at "Disaster City" on the grounds of Texas A\&M University in College Station, Texas.

\section{Funding}

Christopher's research was funded through an NSERC Discovery grant and several Engage grants. 